\title{
An Efficient Hand-Geometry System for Biometric Identifications
}

\author{
Pranoti Das ${ }^{1}$, Sachin Meshram ${ }^{2}$ \\ I, (M.tech student, C/O N.R.Das, New Sarkanda, Bengali Para, Bilaspur, (C.G.), 495001, INDIA ) \\ ${ }_{2}^{2}$ (Assistant Professor, Department of ECE, Chouksey Engineering College, Bilaspur (C.G.), 495004, INDIA)
}

\begin{abstract}
In this paper we propose an efficient hand geometry based system to recognize individuals. Hand geometry used in this verification system utilizes some hand geometry features which cannot vary with small variation of palm position. In this work, users can place their hands freely without the need for pegs to fix the hand placement. This reduces the inconvenience to the users. Our system consists of a database where all the information about the authenticated user is stored. It extracts the features from a test image and compares it with the stored information on the database. The results are encouraging and confirm the utility of our system.

Keywords: Biometrics, Hand-Geometry, Identification, Robust, Verification
\end{abstract}

\section{Introduction}

Biometric is gaining more privilege in recent years. It involves identifying a user based on his physiological or behavioral traits. The conventional authentication systems which are knowledge-based have various drawbacks. In these systems, the users have to memorize lots of password and token-based system having own ID cards for ATM booths, laptop computers and cellular phones. The shortcoming of the above methods is ID cards can be lost or duplicated; passwords can be forgotten or shared. But the solution to the above problem is using physiological or behavioral characteristics or biometric which is more secure, reliable and user friendly. [1]

There are various biometric system based on different characteristics and parts of the human body. Some of the commonly used biometric identification systems are based on face, fingerprint, iris and hand. This paper is based on hand geometry which is one of the efficient biometric identification system. [2]

\subsection{Hand Geometry}

Among all the widely used biometric measurement systems, each has its strengths and limitations and accordingly they are used for particular application. The acceptability is one of the important parameter for any biometric system. In high security applications, biometrics like face recognition and iris system are not acceptable as face is mostly prone to aging and a special illumination setup is needed by iris or retina-based identification systems. The proposed hand-geometry system is solution to the above mentioned problems.

Hand geometry-based verification system is also very efficient for various other reasons. In our system, the data required to uniquely identify a user is very less, so a large number of templates can be easily stored. Hand geometry systems will generally only require a template size of 10 bytes. In this system, the failure to enroll rate is very low. It is easy to use and non-intrusive in nature. [3]

The prior work related to hand geometry systems are based on analysis of palm prints in 2D mode. The pegs are used for proper placement of hands but sometimes they create deformities in the test images.

In our approach, we use peg-free analysis and the images are acquired from conventional webcam, so it is robust for low quality images also. Our system is multimodal in nature i.e. the $3 \mathrm{D}$ feature of hand can be extracted by this system.

\section{Methodology}

The hand-geometry based identification system consists of five important modules; image acquisition, image preprocessing, feature extraction, matching and decision.

\subsection{Image Acquisition}

The first step in hand geometry biometric system is image acquisition. The image acquisition system which we have designed comprises of a conventional digital camera or webcam and a light source. The captured images are stored in various formats such as .jpeg, .tiff, .gif and bmp on the computer for possible image processing.

\subsection{Image Preprocessing}

The second step is image preprocessing module which prepares the hand image for later analysis. As the hand image is captured through digital camera, it is a colored image. For digital image processing it is 
necessary to convert it into gray scale image. An image of this nature varies from blank at the weakest intensity to white at the strongest. The noise removal is done by using wiener filter. Edge detection of image is done at this stage which localizes the pixel intensity transitions. Detecting edges of an image also filters out useless information while preserving the important structural properties in a hand image.

The block diagram of hand-geometry biometric identification system is as shown below:

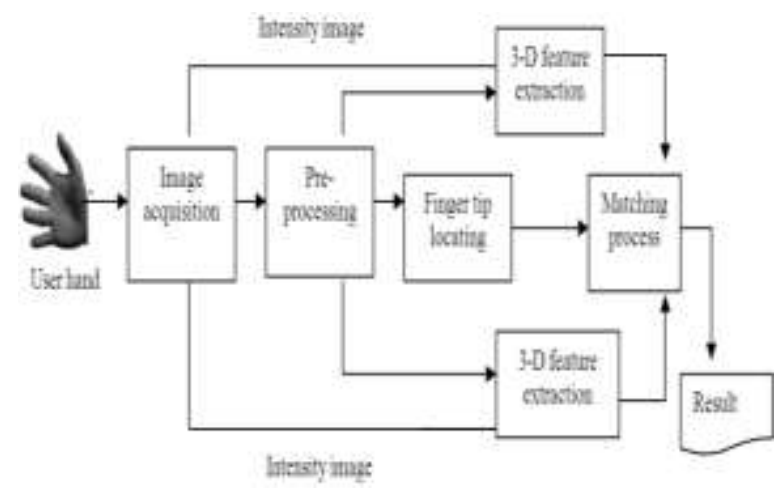

Fig. Block Diagram

\subsection{Feature Extraction}

The third module of our hand-geometry biometric system is feature extraction. It is a special form of dimensionality reduction which transforms the input data into the set of features. In our system there is no peg to fix the placement of hand and as it is a multimodal system, users can place their hands in various positions. So, some of the "landmark points" have to be located which include fingertips and valley points. The reference position is fixed and through it the distance between landmark points and reference points is measured by using Euclidean Distance: [4]

$$
\mathrm{D}=\sqrt{ }\left(\mathrm{x}-\mathrm{x}_{\mathrm{r}}\right)^{2}+\left(\mathrm{y}-\mathrm{y}_{\mathrm{r}}\right)^{2}
$$

In our system we also consider the palm creases and palm textures for personal identifications.

\subsection{Matching}

The last module of our hand-geometry biometric system is matching. It determines the degree similarity between the stored feature vector and claimed feature vector. The fixed threshold is used for matching purpose. Only the matches which are above a certain threshold are said to be valid and the others are rejected.

To decide whether the claimer is the claimed person or not, the distance functions are used. In this work, we adopted the absolute distance method: [1]

$$
D_{\mathrm{a}}=\sum_{\mathrm{i}=1}^{\mathrm{d}}\left|\mathrm{Y}_{\mathrm{i}}-\mathrm{F}_{\mathrm{i}}\right|
$$

\subsection{Decision}

The person whose hand image is found closest to the stored database would be the recognized person. The user with minimum value of absolute distance is considered as the recognized user.

\section{Experiments And Results}

In this work, we have used 30 test users between the age group of 24-29 years. the 3D images of both the hand is acquired from each user. It is stored as a users template or feature vectors in the enrollment phase.

In our work, out of 30 user's both hand images, 28 user's are correctly recognized and remaining are rejected. The Equal Error Rate or ERR is also very less in our system and thus the accuracy of the system is about $93 \%$.

\section{Conclusion}

The motive of this work was to design a system a reliable means meeting today's high security concerns both at personal and institutional level. Our hand-geometry biometric system is very robust and user friendly in nature. The achieved results are significant since the system accuracy rate is $93 \%$ with a very small error rate of approximately $7 \%$.

\section{Acknowledgements}

The authors gratefully acknowledge support from Electronics and telecommunication Engineering Department, Chouksey Engineering College, Bilaspur, (C.G.), and India. We also thank all students and fellows whose samples (hand images) were used in this work. 


\section{References}

[1] Nongluk Covavisaruch, Pipat Prateepamornkul, Puripant Ruchikachorn and Piyanaat Taksaphan, Personal Verification and Identification Using Hand Geometry, ECTI Transactions on Computer and Information Technology, Vol.1,No.2, 2005,134-140

[2] Anil K. Jain, Arun Ross and Sharath Pankanti, A Prototype Hand Geometry-based Verification System, Proc. of $2^{\text {nd }}$ international Conference on Audio and Video-based Biometric person Authentication (AVBPA), Washington D.C.,1999,166-171

[3] Vivek Yadav, Design of a Hand Geometry Based Verification System, Thapar University, Patiala, India, 2010

[4] J.Jobin, Joseph Jiji, Y.A.Sandhya, Saji.Soni, P.L.Deepa, Palm Biometrics Recognition and Verification System, International Journal of advanced Research in Electrical Electronics and Instrumentation Engineering,Vol.1,Issue 2,2012, $41-48$

[5] Jugurta Montalvao, Lucas Molina and Janio Canuto, Robust Hand Image Processing for Biometric Applications, Springer-Verlag London Limited, 2010,397-407

[6] D. Sampada, V.H.Patil, Person Identification using Peg Free Hand Geometry Measurement, international Journal of Engineering Science and Technology (IJEST), Vol.4, No.6, 2012 